\title{
Measuring biting behavior induced by acute stress in the rat
}

\author{
J.L. Zeredo ${ }^{1,4}$, Y. Kumei ${ }^{3}$, T. Shibazaki ${ }^{2}$, N. Yoshida ${ }^{2}$, K. Toda ${ }^{1}$ \\ ${ }^{1}$ Division of Integrative Sensory Physiology, ${ }^{2}$ Division of Orthodontics and Dentofacial \\ Orthopedics, Graduate School of Biomedical Sciences, Nagasaki University, Nagasaki; and \\ ${ }^{3}$ Section of Biochemistry, Department of Hard Tissue Engineering, Graduate School, Tokyo \\ Medical and Dental University, Tokyo, Japan; and ${ }^{4}$ Universidade de Brasília, Brasilia, Brazil.
}

Running head: Rat biting behavior in acute stress

Correspondence to:

Jorge L Zeredo, DDS, PhD. Universidade de Brasília, Campus Universitário Darcy Ribeiro,

Brasília, DF, CEP 70910-900, Tel: (55 61) 3307 2022, e-mail: jllzeredo@gmail.com 


\section{Abstract}

Previous reports indicate that biting behavior is enhanced in rats that are subject to acute stress.

Several methods have been proposed to study this phenomenon, one of which is the electromyography (EMG) of the jaw-muscles. In this study, we compared the total EMG

activity with the EMG activity related to biting behavior, as determined by video monitoring,

before and after restraint stress. Wistar albino rats (150 g) were subject to surgery for electrode

placement 48 hours before measurements. The EMG activity of the masseter muscle on one side

was recorded for 10 minutes before and immediately after 30 minutes of restraint stress.

Restraint stress increased jaw-muscle EMG activity; although some of the activity was related to

behaviors other than biting. Recording EMG activity of jaw-muscles is useful for measuring the

stress response in rats, but careful video monitoring is needed if biting behavior is to be studied in

particular.

(100 to 150 words; current: 149 words)

Keywords: animal behavior, stress, electromyography, masseter muscle, rat. 


\section{Introduction/background}

Emotional stress is known to induce biting behavior in rats, a phenomenon that may be an analogue of bruxism, tooth clenching, and nail biting in humans. In rats, this behavior has been termed purposeless, or "empty" chewing. Although the neurophysiology of these stereotypies in animals or humans is poorly understood, it is widely accepted that one underlying mechanism could be the increase in neuromotor excitability of rhythmical mandibular movements (e.g: Lavigne, Kato, Kolta, \& Sessle, 2003). Such excitability may be triggered by stress or anxiety; therefore, biting behavior has been proposed as a valid parameter to assess stress response in rats (Byrd, 1997; Pohto, 1979; Rosales et al., 2002). Abnormal biting behavior is also seen in oromandibular dystonia, a condition that can be provoked by pharmacological agents or caused by abnormalities in neurons of the basal ganglia (Clark, Koyano, \& Browne, 1993; Tamminga, Dale, Goodman, Kaneda, \& Kaneda, 1990). Therefore, it would be useful to develop a simple and reliable method to quantitatively study biting behavior in the rat. The purpose of this study was to determine the applicability of electromyography for the automated measurement of the rat's biting behavior in response to acute stress. 


\section{Methods}

The methods described here follow the ethical guidelines and received approval by the Animal Welfare Committee of Nagasaki University. Under barbiturate anesthesia, Wistar albino rats weighing $150 \mathrm{~g}$ were used (n=12). Electromyography (EMG) electrodes were implanted into the masseter muscle on one side. EMG electrodes consisted of two stainless steel wires (Teflon coated, $125 \mu \mathrm{m}$ in diameter; A-M Systems, Carlsborg, USA) insulated except for $3 \mathrm{~mm}$ at the tip. One end of each wire was passed through the muscle $3 \mathrm{~mm}$ away from the other with the help of a 25G disposable needle; the other end was passed from under the skin toward the top of the head and soldered to a connector, which was fixed to the skull with dental acrylic. Penicillin G was administered (30,000 IU, i.m., Dainippon Sumitomo Pharma, Tokyo, Japan). After 48 h, the rats' biting behavior was recorded before and after acute restraint stress. The rats' biting behavior was assessed by the masseter muscle EMG and by video monitoring of the freely-moving animals for 10 minutes. Restraint stress consisted of placing the animals inside a restraining cylinder for 30 minutes. During recordings the animal was connected to the EMG apparatus by a flexible cable attached to its head (Fig. 1). The cable was kept elevated to avoid entanglement without restraining animal movement. The EMG signal was passed through a differential amplifier (DAM-80, WPI, Sarasota, USA) and filtered, digitalized, and stored in a computer. 
Data analysis

The EMG signal of each rat was analyzed with the aid of the Spike2 software (CED, Cambridge, UK). Waveforms with amplitude greater than $2 \mathrm{SD}$ of the baseline were considered as indicative of muscle activity. The total time of muscle activity was measured. Then, the EMG signal was matched with the video image and the EMG activity not related to empty chewing was removed. Paired comparisons were made by the non-parametric Wilcoxon Signed Rank test, where $\mathrm{p}<0.05$ was considered significant.

\section{Results}

The animals recovered well from surgery and did not show signs of infection throughout the experimental period. The recorded EMG activity was greater after restraint as compared to the activity in the same animal before restraint (Fig 2). In 5 of 12 rats (about 40\%) parts of the recorded EMG activity were associated with grooming behavior. There was statistically significant difference between the total time of muscle activity and the time of muscle activity that matched biting behavior (on visual inspection) after restraint (Fig. 3). However, in either measurements (total EMG and filtered EMG) the values after restraint were significantly greater than before restraint $(\mathrm{p}<0.05$ in the Wilcoxon Signed Rank test). 


\section{Discussion}

Most of the previous studies that analyzed biting behavior in the rat used visual (video) inspection (e.g.: Tamminga et al., 1990). A trained observer measures biting behavior in terms of seconds of empty chewing or number of bursts of chewing activity. Indeed, quantification of tooth grinding or biting behavior is difficult to automate as it must be dissociated from other orofacial activities such as purposeful biting, licking, or grooming. A previous study used electromyography of jaw muscles, and filtered out biting activity by counting only cyclic bursts of large amplitude and longer duration (Rosales et al., 2002). Other studies also found the same relationship between EMG activity in the masseter muscle and empty chewing movements (Shoji, Bruce, \& Siu, 1994); however, in this study we found instances of biting behavior associated with EMG activity of smaller amplitude as well. Such discrepancy may be caused by minor differences in electrode configuration and placement in each study. Other methods, such as motion capturing, may not be feasible unless the animal body is considerably restrained. One company that we contacted at the site of the Measuring Behavior 2008 conference claimed to have developed an apparatus capable of automatically quantifying purposeless chewing in rats with over $80 \%$ correlation with observer-registered measurements (Laboras, Metris BV, The Netherlands); however, we could not find any published articles that used their equipment to measure biting behavior. 
In this study we used EMG signal from one of the major jaw muscles involved in mastication and biting, the masseter muscle. Activity in this muscle was higher after exposure to restraint stress. This method had the advantage of being relatively simple and inexpensive.

Disadvantages include the need for surgery for electrode implantation, which exposes the animals some stress and risk of infection. Visual (video) monitoring showed that oral behaviors other than biting, such as when the animal licks the body during grooming, were included in the EMG signal. Unfortunately, for this reason the method could not be fully automated for the measurement of biting behavior. Nevertheless, both measurements of EMG activity were greater after restrain stress, possibly because the major source of EMG activity not related to biting was grooming behavior, which is also increased by stress (e.g.: Yozgatian et al., 2008). One animal managed to touch the cable connector during grooming; this introduced a few artifacts into the signal, although it could be easily sorted out and removed. Another potential disadvantage for studies of stress-related behavior is that the EMG signal cannot be obtained if the paradigm involves immersion of the animal in water or if cable connection is not feasible (e.g. inside a centrifuge).

Restraint stress caused an increase in biting behavior, which was quantifiable by jaw-muscle 
EMG. It is suggested that biting behavior is a stereotypical component of the stress response in rats. Recording the EMG signal from jaw muscles may be useful in quantifying biting behavior, but careful video monitoring is recommended in order to seek out other oral behaviors.

\section{Acknowledgments}

This work was supported by KAKENHI (No. 19791589 to JLZ, FY2007-2008). 


\section{References}

Byrd, K. E. (1997). Characterization of brux-like movements in the laboratory rat by optoelectronic

mandibular tracking and electromyographic techniques. Arch Oral Biol, 42(1), 33-43.

Clark, G. T., Koyano, K., \& Browne, P. A. (1993). Oral motor disorders in humans. J Calif Dent Assoc, 21(1), 19-30.

Lavigne, G. J., Kato, T., Kolta, A., \& Sessle, B. J. (2003). Neurobiological mechanisms involved in sleep bruxism. Crit Rev Oral Biol Med, 14(1), 30-46.

Pohto, P. (1979). Experimental aggression and bruxism in rats. Acta Odontol Scand, 37(2), 117-126.

Rosales, V. P., Ikeda, K., Hizaki, K., Naruo, T., Nozoe, S., \& Ito, G. (2002). Emotional stress and brux-like activity of the masseter muscle in rats. Eur J Orthod, 24(1), 107-117.

Shoji, Y. N., Bruce, I. C., \& Siu, L. Y. (1994). Electromyographic assessment of non-functional masseter muscle in an awake animal model. Cranio, 12(2), 110-113.

Tamminga, C. A., Dale, J. M., Goodman, L., Kaneda, H., \& Kaneda, N. (1990). Neuroleptic-induced vacuous chewing movements as an animal model of tardive dyskinesia: a study in three rat strains. Psychopharmacology (Berl), 102(4), 474-478.

Yozgatian, J. H., Zeredo, J. L., Hotokezaka, H., Koga, Y., Toda, K., \& Yoshida, N. (2008). Emotional stressand pain-related behaviors evoked by experimental tooth movement. Angle Orthod, 78(3), 
487-494.

Page $\mathbf{1 0}$ of $\mathbf{1 1}$ 


\section{Figure legends}

Figure 1. Rat under observation during EMG recording. Inset: Same rat in the restraining cylinder.

Jaw-muscle activity was measured before and after 30 minutes of stress stimulation in the restraining cylinder.

Figure 2. Samples of EMG tracings. Restraint stress increased jaw-muscle EMG activity; although in some cases some of the activity was related to grooming (horizontal bars) rather than biting behavior.

Figure 3. Jaw-muscle EMG activity before and after restraint stress. Before' and After' are the values filtered for behaviors other than biting. EMG activity was significantly less in the filtered measurement after restraint, but not before restraint. Both measurements (filtered and not filtered) were significantly greater after restraint than before restraint. Asterisk indicate $\mathrm{p}<0.05$ in the Wilcoxon Signed Rank test. 
Figure 1

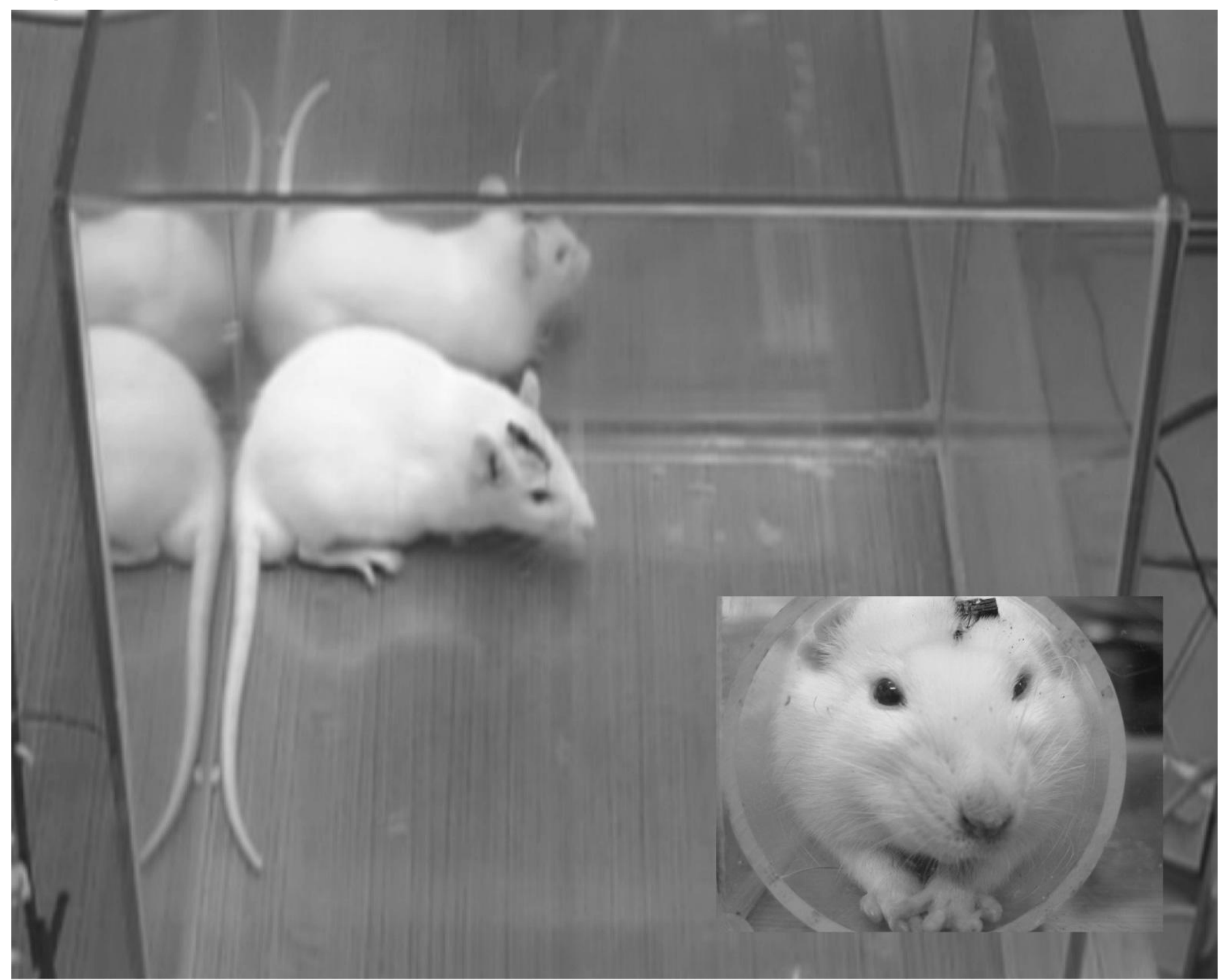


Figure 2

Before restraint

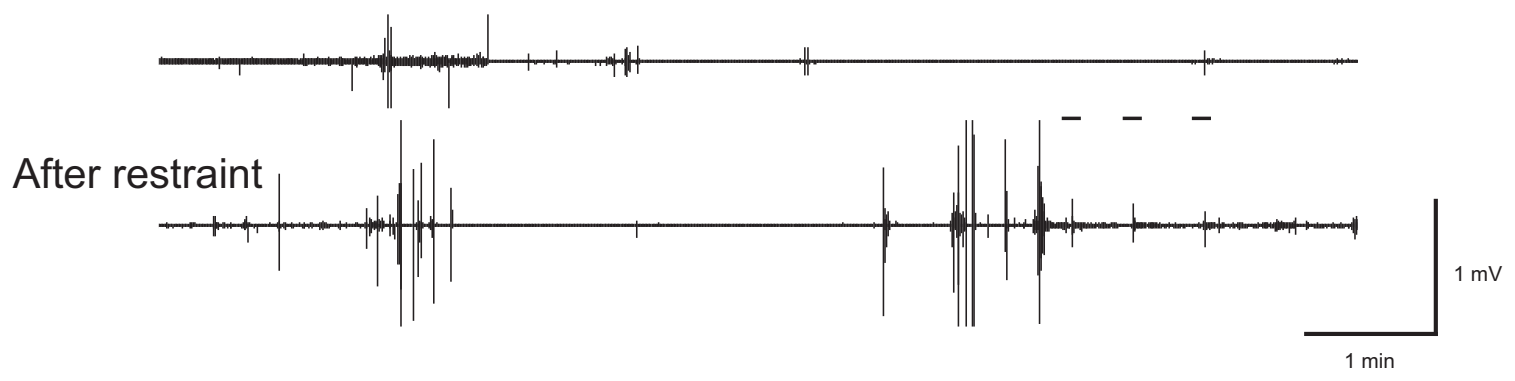


Figure 3

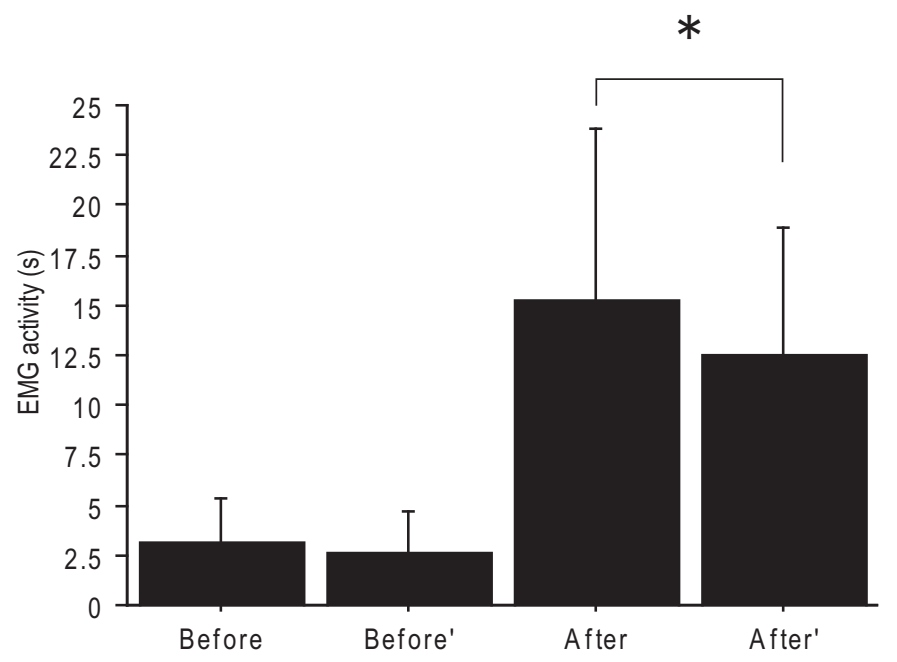

